\section{Manipulation and reversal of the probability of a correct stimulus prediction in a choice reaction task*}

\author{
CHARLES P. WHITMAN and E. SCOTT GELLER $†$ \\ Virginia Polytechnic Institute and State University, Blacksburg, Va. 24061
}

In a two-stimulus, two-response choice reaction time (RT) experiment, the probability of a correct stimulus prediction was controlled: the probability $(\mathrm{P})$ was .70 or .30 for 400 trials or $P$ was .70 or .30 for 200 trials and $1-P$ for the remaining 200 trials. The difference between $\mathrm{RT}$ to correctly predicted stimuli and RT to incorrectly predicted stimuli was greater when $P$ was .70 than when it was .30. When $P$ shifted from .30 to .70 , the effect of prediction outcome increased; the effect of prediction outcome decreased when $\mathrm{P}$ shifted from .70 to .30. Implications for learning in the development of expectancies for predicting correctly are discussed.

Choice reaction time $(\mathrm{RT})$ is shorter to correctly predicted stimuli than to incorrectly predicted stimuli (e.g. Bernstein \& Reese, 1965; Williams, 1966 ) and shorter to more probable stimuli than to less probable stimuli (e.g., LaBerge \& Tweedy, 1964; Laming, 1969). Furthermore, RT studies that examined the effects of both prediction outcome and stimulus probability demonstrated shorter. RT to the more probable stimulus than to the less probable stimulus regardless of the prediction outcome (Geller, Whitman, Wrenn, \& Shipley, 1971; Geller, Whitman, \& Farris, 1972; Hinrichs \& Craft, 1971). Moreover, when Geller et al reversed a frequency imbalance midway through the experimental session, RTs were shorter to the more probable stimulus alternative within 100 trials after the probability reversal. These within-Ss differences in RT implied that particular stimulus expectancies were initially developed and then reversed following the probability change.

Recently, Whitman \& Geller (1972) manipulated the probability of a correct stimulus prediction in a two-choice RT task and found the effect of prediction outcome to increase directly as the probability of a correct prediction increased. The result that $\mathrm{RT}$ to correctly predicted stimuli was an inverse function of the probability of a correct prediction indicated that Ss' expectancy to make a correct prediction increased as the probability of a correct prediction increased. The present choice RT

*This research was partially supported by the Small Grants Program of the College of Arts and Sciences, Virginia Polytechnic Institute and State University.

tRequests for reprints should be sent to E. Scott Geller. Department of Psychology, Virginia Polvtechnic Institute and State Universitv, Blicksburg, Va. 24061. experiment reversed the probability of a correct stimulus prediction after 200 predictions and compared pre- and postreversal effects of prediction outcome on reaction latencies. Whereas choice RTs implied changes in reversal of stimulus probabilities (Geller et al, 1971, 1972), the present experiment was designed to, determine if RTs will indicate chainges in Ss' expectancy to make a correct stimulus prediction following a reversal of the probability of a correct prediction.

\section{SUBJECTS}

The 80 Ss were volunteers from introductory psychology classes at Virginia Polytechnic Institute and State University and had not previously participated in a choice RT experiment. The only three Ss who made anticipatory or choice errors on more than $5 \%$ of the RT trials were replaced.

\section{APPARATUS}

Prior to each of 400 stimulus presentations in a two-stimulus, two-response choice reaction task, Ss made stimulus predictions by pressing one of two prediction switches and then verbally indicating their choice. The two stimuli were verbally labeled "up" and "down" and were, respectively, the symbols $L$ and $\Pi$ as presented by a $1.5 \times 2.5 \mathrm{~cm}$ digital readout. The two response mechanisms were microswitch triggers encased in left- and right-hand handles.

\section{DESIGN}

Twenty Ss were assigned randomly to each of four experimental conditions. For Group 70/30, the probability of a correct stimulus prediction was .70 during the initial 200 trials and .30 during the subsequent 200 trials, while for Group $70 / 70$, the probability of a correct prediction was .70 for all 400 trials. The $S$ s in Group $30 / 70$ made correct stimulus expectancies following a stimulus predictions on $30 \%$ of the initial 200 trials and then made correct predictions on $70 \%$ of the remaining 200 trials. The Ss in Group $30 / 30$ made correct predictions on $30 \%$ of all 400 trials. Thus, the design was a between-Ss factorial of 2 (preshift probabilities of a correct prediction, .70 or .30) by 2 (postshift probabilities of a correct prediction, .70 or .30$)$.

A .70 binary distribution was determined by filtering a uniform random number generator on an IBM 370 computer system and was punched on paper tape. On each trial, relay equipment sampled the paper tape via a tape reader and determined whether the stimulus predicted or the alternative stimulus would occur. \section{PROCEDURE}

Each trial began when E presented a horizontal segment of the digital readout as a signal for $S$ to make a stimulus prediction by pressing one of two prediction levers. Following a prediction, E sounded a brief warning buzzer which preceded the stimulus presentation by a variable time interval of between .5 and $1.5 \mathrm{sec}$. The S's choice response turned off the stimulus and stopped a digital millisecond timer. The RT was manually recorded before the presentation of the prediction signal that initiated a new trial. The total time per trial was approximately $8 \mathrm{sec}$. Ten Ss in each of the four groups responded with the right trigger to $\sqcup$ and with the left trigger to $\Pi$, while the remaining $10 \mathrm{Ss}$ used the reverse $\mathrm{S}-\mathrm{R}$ relationship.

\section{RESULTS}

The latencies of correct identifications for each $S$ were categorized according to prediction outcome and consecutive 100-trial blocks. The data illustrated in Fig. 1 are the averages of the Ss' means for each category of each group.

As evident in Fig. 1, RT to correctly predicted stimuli was shorter than RT to incorrectly predicted stimuli and the effect of prediction outcome was greater when the probability of a correct prediction was .70 than when it was .30 . The overall analysis of variance was 2 (preshift probability of a correct prediction, .30 or .70 ) by 2 (postshift probability of a correct prediction, .30 or .70) by 2 (prediction outcome, correct or incorrect) by 4 (trial blocks of 100). Neither the main effects of preshift probability of a correct prediction nor those of postshift probability of a correct prediction were reliable (both $\left.F_{s}<1.6\right)$. The main effects of prediction outcome and trial block were significant $[F(1,76)=102.9$ and $\mathrm{F}(3,228)=38.9$, respectively, both ps $<.001]$. The prediction outcome 


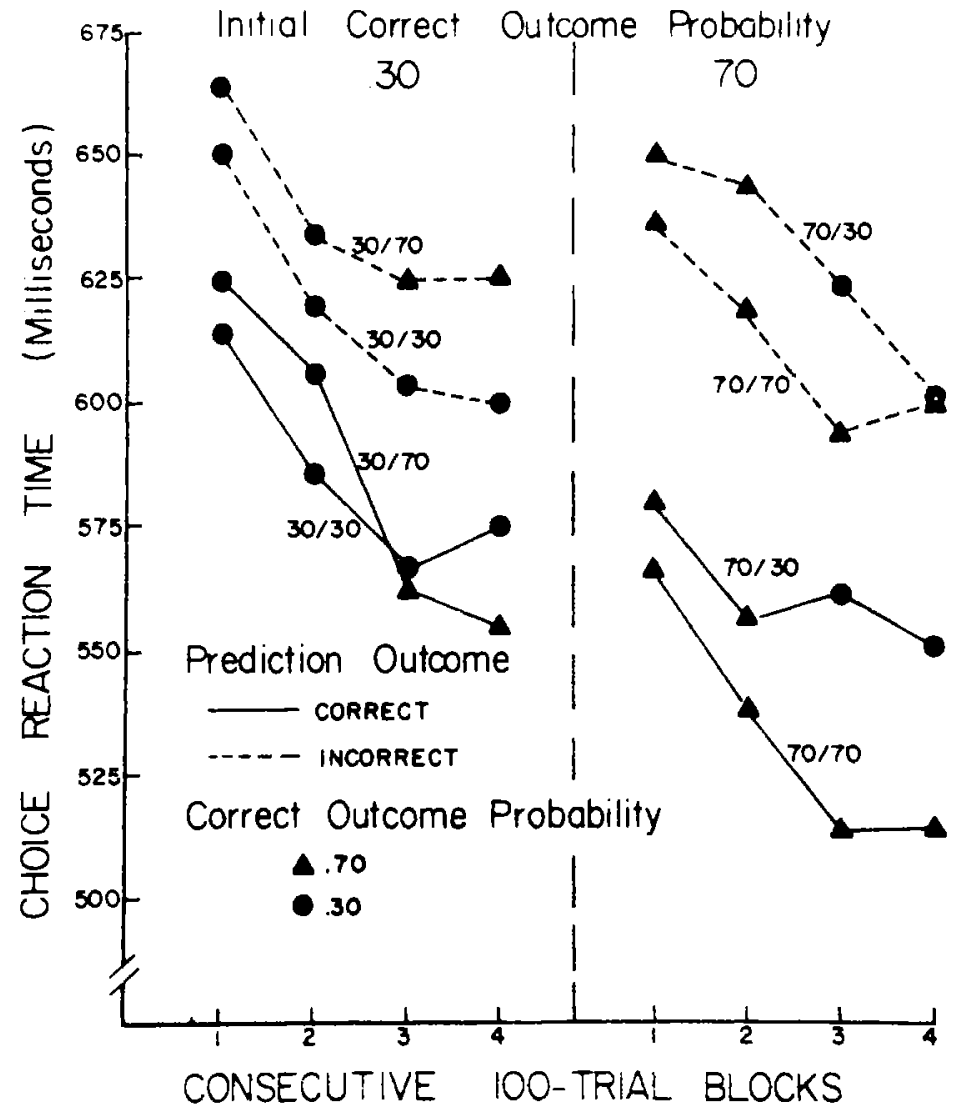

Fig. 1. Choice reaction time as a function of prediction outcome, the probability of a correct prediction, and consecutive 100 -trial blocks.

variable was involved in three reliable interactions: Prediction Outcome by Preshift Probability of a Correct Prediction $[F(1,76)=8.36, p<.01]$ Prediction Outcome by Preshift Probability of a Correct Prediction by Trial Block $[\mathrm{F}(3,228)=5.08$, $\mathrm{p}<.005$ ], and Prediction Outcome by Postshift Probability of a Correct Prediction by Trials $[\mathrm{F}(3,228)=9.62$, $p<.001]$. None of the remaining interactions was significant (all Fs $<1.6$ ).

In order to study the nature of the third-order interactions, a 2 (prediction outcome) by 4 (trial block) analysis of variance was computed for each group. In each of the four analyses, there were reliable main effects of prediction outcome and of trial block (all ps $<.05$ ). Between-group effects were indicated by the differential interactions obtained. For Groups 30/30 and $70 / 70$, in which the probability of a correct prediction did not change, the Prediction Outcome by Trial Block interactions were not significant (both ps $>.10$ ), while for Groups 30/70 and $70 / 30$, these interactions were reliable $[F(3,57)=6.51$ and $F(3,57)=5.83$, respectively, both ps $<.005]$. Thus, the effect of prediction outcome on choice RT did not change as a function of trials when the probability of a correct prediction was held constant, but the magnitude of the effect of prediction outcome shifted reliably when the probability of a correct prediction was reversed.

\section{DISCUSSION}

On an empirical level, the results of the present experiment replicated the findings of previous choice RT studies The observation that RT to correctly predicted stimuli was shorter than RT to incorrectly predicted stimuli was made previously by several 1965; Geller et al, 1971, 1972; Hinrichs \& Craft, 1971). Moreover, Whitman \& Geller (1972) also observed a reliably greater effect of prediction outcome when the probability of a correct prediction was .70 than when it was .30 .

To interpret the influence of the probability of a correct stimulus prediction on the prediction outcome effect, Whitman \& Geller (1972) assumed that Ss' expectancy to make a correct prediction was a direct function of the probability of a in which Ss made stimulus predictions. investigators (e.g., Bernstein \& Reese, correct prediction. Furthermore, RT to correctly predicted stimuli was a decreasing function of the expectancy and RT to incorrectly predicted stimuli was an increasing function of the expectancy. Thus, an increase in the probability of a correct stimulus prediction increased Ss' expectancy to predict correctly and resulted in a prediction outcome effect of greater magnitude.

During the initial 200 trials of the present experiment, the greater effect of prediction outcome observed when the probability of a correct prediction was .70 than when it was .30 implied also that Ss' degree of expectancy to make correct predictions varied between conditions. In addition, following the shift in the probability of a correct prediction, mean RTs of Group 30/70 exhibited a marked increase in the prediction outcome effect and implied that these Ss increased their expectancy for the predicted stimulus as a function of the greater frequency of correct predictions. Conversely, when the probability of a correct prediction was reversed from .70 to .30 , there was a reliable decrease in the prediction outcome effect, supporting a notion that Ss of Group 70/30 learned to reduce their level of expectancy for a predicted stimulus subsequent to a lowering of their prediction success.

Recent choice RT studies which reversed the frequency imbalance of two stimulus alternatives (Geller et al, 1971,1972 ) found shorter mean RT to the more probable stimulus within 100 trials following the probability reversal and thus concluded that stimulus expectancies that were learned during the preshift trials were reliably altered by the probability change. The influence of reversing the probability of a correct prediction on the prediction outcome effect observed in the present study was analogous to the observed consequence of reversing stimulus frequency on the probability effect in the sense that the results of both experiments implicated changes in learned expectancies.

\section{REFERENCES}

BERNSTEIN, I. H., \& REESE, C. Behavioral hypotheses and choice reaction time. Psychonomic Science, $1965,3,259-260$.

GELLER, E. S., WHITMAN, C. P., WRENN, R. F., \& SHIPLEY. W. G. Expectancy and discrete reaction time in a probability reversal design. Journal of Experimental Psychology, 1971, 90, 113-119.

GELLER, E. S.. WHITMAN, C. P., \& FARR IS, j. C. Probability discrimination indicated by stimulus predictions and reaction speed: Effects of S-R compatibility. Journal of Experimental Psychology. 1972, 93, 404-409.

HINRICHS, J. V.. \& CRAFT, J. L. Verbal 
expectancy and probability in two-choice reaction time. Journal of Experimental Psychology, 1971, 88, 367-37i.

LABERGE, D. \& TWEEDY, J R. Presentation probability and choice time. Journal of Experimental Psychology, $1964,68,477-481$.
LAMING, D. R. J. Subjective probability in choice-reaction experiments. Journal of $M$ athematical Psychology, 1969, 6, 81-120.

WHITMAN, C. P., \& GELLER, E. S. Prediction outcome probabilities as determinants of choice reaction time.
Perception \& Psychophysics, in press.

WILLIAMS, J. A. Sequential effects in disiunctive reaction time: Implications for decision models. Journal of
Experimental Psychology, 1966, 71 , 665-672. 